\title{
Validating the role of the Australian
}

Shea J. Andrews ${ }^{1,2^{*}}$ (D), Ranmalee Eramudugolla ${ }^{2}$, Jorge I. Velez ${ }^{1,3,4}$, Nicolas Cherbuin ${ }^{2}$, Simon Easteal ${ }^{1}$ and Kaarin J. Anstey ${ }^{2}$

\begin{abstract}
Background: The number of people living with dementia is expected to exceed 130 million by 2050, which will have serious personal, social and economic implications. Employing successful intervention and treatment strategies focused on disease prevention is currently the only available approach that can have an impact on the projected rates of dementia, with risk assessment being a key component of population-based risk reduction for identification of at-risk individuals. We evaluated a risk index comprising lifestyle, medical and demographic factors (the Australian National University Alzheimer's Disease Risk Index [ANU-ADRI]), as well as a genetic risk score (GRS), for assessment of the risk of progression to mild cognitive impairment (MCl).

Methods: The ANU-ADRI was computed for the baseline assessment of 2078 participants in the Personality and Total Health (PATH) Through Life project. GRSs were constructed on the basis of 25 single-nucleotide polymorphisms previously associated with Alzheimer's disease (AD). Participants were assessed for clinically diagnosed $\mathrm{MCl}$ and dementia as well as psychometric test-based $\mathrm{MCl}$ (MCl-TB) at 12 years of follow-up. Multi-state models were used to estimate the odds of transitioning from cognitively normal (CN) to $\mathrm{MCl}$, dementia and $\mathrm{MCl}-\mathrm{TB}$ over 12 years according to baseline ANU-ADRI and GRS.

Results: A higher ANU-ADRI score was associated with increased risk of progressing from $\mathrm{CN}$ to both $\mathrm{MCl}$ and MCI-TB (HR 1.07 [95\% Cl 1.04-1.11]; 1.07 [1.04-1.09]). The GRS was associated with transitions from CN to dementia (HR 4.19 [95\% Cl 1.72-10.20), but not to MCl or MCl-TB (HR 1.05 [95\% Cl 0.86-1.29]; 1.03 [0.87-1.21]). Limitations of our study include that the ethnicity of participants in the PATH project is predominately Caucasian, potentially limiting the generalisability of the results of this study to people of other ethnicities. Biomarkers of AD were not available to define $\mathrm{MCl}$ attributable to AD. Not all the predictive variables for the ANU-ADRI were available in the PATH project.

\footnotetext{
* Correspondence: shea.andrews@anu.edu.au

${ }^{1}$ John Curtin School of Medical Research, Australian National University, Canberra, Australia

${ }^{2}$ Centre for Research on Ageing, Health and Wellbeing, Research School of Population Health Australian National University, The Australian National University Florey, Building 54, Mills Road, Acton ACT 2601, Canberra, Australia Full list of author information is available at the end of the article
} 
(Continued from previous page)

Conclusions: In the general population, the ANU-ADRI, comprising lifestyle, medical and demographic factors, is associated with the risk of progression from $\mathrm{CN}$ to $\mathrm{MCl}$, whereas a GRS comprising the main AD risk genes was not associated with this risk. The ANU-ADRI may be used for population-level risk assessment and screening.

Keywords: Alzheimer's disease, Cognitive aging, Mild cognitive impairment (MCl), Cohort studies, Risk factors in epidemiology, Multi-state models

\section{Background}

Accurate risk assessment for cognitive impairment and dementia is increasingly important, given the current lack of effective disease-modifying treatments for Alzheimer's disease (AD) and other dementias. Risk assessment tools may be used in both pharmacological and non-pharmacological trials, in clinics, and for population-level screening to guide risk reduction strategies $[1,2]$. Validated risk assessment tools that can be administered at very low cost provide methods for clinicians in low-income countries and regions to assess dementia risk and apply prevention strategies. Given current projections of increasing dementia prevalence, there is an urgent need for validated risk assessment tools that have been evaluated in well-characterised samples over long time periods [3]. However, to our knowledge, established dementia risk tools [4] have not been evaluated for assessment of risk of mild cognitive impairment (MCI), which is a key target group for secondary prevention and pharmaceutical trials. Using a recently developed risk tool for $\mathrm{MCI}$ formulated in the Mayo Clinic Study of Aging, researchers found that a basic risk score composed of general demographic (e.g., age, education, marital status) and clinical (e.g., diabetes, hypertension, body mass index $[\mathrm{BMI}]$ ) features had a c-statistic of 0.60. An augmented version containing additional variables typically collected in clinical and neurological examinations (e.g., gain speed, anxiety, Clinical Dementia Rating Sum of Boxes) had a c-statistic of 0.70 [5]. Further evaluation of this model in an independent cohort is required.

Recently, there has also been increasing interest in the evaluation of genetic risk scores (GRSs) for AD and dementia, which have been associated with the development of $A D$ and incident MCI [6-9], though they have limited utility in predicting $\mathrm{AD}$ beyond that attained with basic demographic variables such as age, sex and education $[7,10,11]$. The number of studies assessing the association of AD GRS with progression between cognitive states is limited and the findings mixed. These studies include reports of a significant association between GRS and progression from cognitively normal $(\mathrm{CN})$ to either MCI or late-onset Alzheimer's disease (LOAD) with a c-statistic of 0.684 (HR 1.29 [95\% CI 1.19-1.39]) [10]. Regarding the conversion from MCI to LOAD, one study found that participants harbouring 6 or more $\mathrm{AD}$ risk alleles progressed to AD twofold (HR 1.89 [95\% CI 1.01-3.56]) more rapidly than those with only 6 alleles [12], whereas researchers in a second study observed that an AD GRS composed of 19 loci was associated with the conversion to dementia (HR 1.59 [95\% CI 1.23-2.05]), but only when apolipoprotein $\mathrm{E}(A P O E)$ was included in the risk score [9]. Conversely, researchers in a third study found no association between progression to dementia from MCI using an AD GRS composed of 18 loci [13].

Our study had two aims. First, we sought to evaluate the association of a non-genetic risk index with the progression from $\mathrm{CN}$ to cognitive impairment. Our measure [14] is a self-report risk index (the Australian National University Alzheimer's Disease Risk Index [ANU-ADRI]) that has been externally validated in three cohorts of older adults in which it was found to be predictive of $\mathrm{AD}$ and dementia [15]. The second aim of the present study was to compare the ANU-ADRI with a GRS. We examined the association between cognitive impairment and the ANU-ADRI and a LOAD GRS, as assessed using a clinical criterion for MCI or dementia and psychometric test-based criteria for MCI (MCI-TB) in a community-based cohort of older adults. We first used a Cox proportional hazards model to investigate the association between the ANU-ADRI and a LOAD GRS and incident $\mathrm{MCI} /$ dementia, and then we extended this model using multi-state models (MSMs) to account for backward transitions between cognitive states (i.e., cognitive recovery) and competing risks (i.e., dementia and death).

\section{Methods}

\section{Participants}

Participants were community-dwelling adults residing in the City of Canberra, Australia, or in the neighbouring town of Queanbeyan who had been recruited into the Personality and Total Health (PATH) Through Life project, a longitudinal, population-based study of health and well-being in adults. Cohorts aged 20-24 (20+), 40-44 $(40+)$ and 60-64 (60+) years at baseline were assessed at 4-year intervals for a total of 12 years. The background and procedures for the PATH study are described elsewhere [16]. Written informed consent was obtained from all participants. This study was approved by the 
human research ethics committee of The Australian National University.

In this study, we used data from the $60+$ cohort with interviews conducted in 2001-2002 $(n=2551)$, 2005$2006(n=2222), 2009-2010(n=1973)$ and $2014-2015$ $(n=1645)$. Individuals were excluded if their ethnicity was not Caucasian $(n=107)$ or if they had a selfreported history of stroke, transient ischemic attack, epilepsy, brain tumours or brain infection $(n=381)$.

\section{ANU-ADRI risk assessment based on demographic, lifestyle and medical risk factors}

The development of the ANU-ADRI and the methodology underlying its computation have been described previously [15]. The ANU-ADRI can be computed on the basis of up to 15 predictive variables, 11 of which are available in PATH, including age (self-report), sex (self-report), alcohol consumption (calculated according to National Health and Medical Research Council 2001 guidelines [17] using number of drinks per week, with light to moderate intake in males being $0.25-20.5$ drinks per week and in females being 0.25-13.5 drinks per week), education (self-reported number of years of education), diabetes (self-reported history of diabetes), depression (assessed using the Patient Health Questionnaire [PHQ-9] [18] following the coding algorithm provided in the PHQ-9 instruction manual, with a score $>10$ used as a cut-off), traumatic brain injury (self-reported history of traumatic brain injury with loss of consciousness), smoking (self-reported smoking status as current smoker, past smoker or never smoker), social engagement (constructed from four domains for marital status, size of social network, quality of social network, level of social activities; a fifth domain for living arrangements was not available in PATH and thus was computed pro rata as the average of the above-mentioned social engagement variables), physical activity (combined self-reported number of hours performing mild, moderate and vigorous activities, weighted by multiples of 1,2 and 3, respectively [19]), cognitively stimulating activities (assessed as the number of cognitive activities undertaken in the last 6 months, comprising reading, writing, playing games or attending cultural events), and BMI (weight divided by height squared, expressed in kilograms per square meter). No data were available for the remaining three predictive variables: cholesterol, fish intake and pesticide exposure. The ANUADRI is still predictive of the development of dementia, even when a subset of variables is used [15]. Values for predictive variables included in the ANU-ADRI for PATH were selected from baseline measurements or the first occasion on which the variables were measured. To facilitate interpretation, a constant of +13 was added to the ANUADRI to change the range to from -13 to +19 to $0-32$.

\section{Genotyping and genetic risk score}

The most significant LOAD risk single-nucleotide polymorphisms (SNPs) identified via genome-wide association studies (GWASs) [11, 20-24] from 23 loci (ABCA7, BIN1, CD2AP, CD33, CLU, CR1, EPHA1, MS4A4A, MS4A4E, MS4A6A, PICALM, HLA-DRB5, PTK2B, SORL1, SLC24A4-RIN3, DSG2, INPP5D, MEF2C, NME8, ZCWPW1, CELF1, FERMT2 and CASS4) were genotyped using TaqMan OpenArray assays (Life Technologies, Carlsbad, CA, USA) as previously described $[25,26]$, in addition to the two SNPs defining the $A P O E$ alleles, which were genotyped using TaqMan assays as previously described [27]. Using these LOAD risk SNPs, an explained variance-weighted genetic risk score (EVGRS) [28] was constructed, which is the sum of all the risk alleles across the individual, weighted by minor allele frequency (MAF) and the OR associated with LOAD. The EV-GRS is calculated according to the following formula:

$$
\mathrm{EV} \_\mathrm{GRS}=\sum_{i=1}^{I}\left(\log \left(O R_{i j}\right) \sqrt{2 M A F_{i j}\left(1-M A F_{i j}\right)}\right) * G_{i j}
$$

for the $i$ th patient, where $\log \left(O R_{i}\right)=$ the OR for the $j$ th SNP, $M A F_{i j}=$ the MAF for the $j$ th SNP, and $G_{i j}=$ the number of risk alleles for $j$ th SNP. Individuals with missing genetic data were excluded $(n=240)$. We weighted the LOAD SNPs using the previously reported OR for LOAD and by the MAF for the CEU reference population (Utah residents with Northern and Western European ancestry; see Additional file 1: Table S1). The EV-GRS was transformed into a z-score.

\section{Screening and clinical assessment}

The screening and clinical assessment methods at waves $1-3$ are described elsewhere $[29,30]$ and are briefly summarised here. At each wave, the same predetermined cutoff derived from a battery of cognitive tests was used for inclusion of participants in a sub-study on mild cognitive disorders and dementia. Participants from the full cohort were selected for clinical assessment if they had any of the following: (1) a Mini Mental State Examination (MMSE) [31] score <25; (2) a score below the fifth percentile score on immediate or delayed recall of the first list of the California Verbal Learning Test [32]; or (3) a score below the fifth percentile on two or more of the Symbol Digit Modalities Test (SDMT) [33], Purdue Pegboard with both hands [34] or Simple Reaction Time [35]. At wave 4, participants were selected for review if they met any of the following criteria: (1) MMSE score $<25$ or $\leq 2.5^{\text {th }}$ percentile on one or more cognitive test, (2) previous diagnosis at waves $1-3$, (3) subjective decline $\geq 25$ on the Memory and Cognition Questionnaire (MACQ) or (4) decline in MMSE score $\geq 3$ points. 
The criteria for the clinical assessment for cognitive impairment at waves 1-3 has been published by our group elsewhere [30]. They involved a structured clinical assessment for dementia conducted by one of two physicians, a neuropsychological assessment, and the Clinical Dementia Rating [36], which were used together to formulate a consensus diagnosis.

Owing to the large number of participants screened for review at wave 4 , diagnosis was based on neurologist review of interview data as outlined below and in Fig. 1. For each of the 1644 participants with interview data at wave 4, assessment data were screened for signs of decline on the basis of the following criteria (screen 1): a previous diagnosis of a cognitive disorder at waves 1,2 or 3 or either evidence of cognitive impairment on the MMSE ( $\leq 24)$ or performance on one or more cognitive tests $\leq 6.7$ th percentile at wave 4 (immediate recall task, delayed recall task, SDMT, F words, A words, Boston Naming Test, Simple Response Time task, Choice Response Time task, Purdue Pegboard dominant, Purdue Pegboard non-dominant, Purdue Pegboard both, Digit Span Backward, Trail Making Test B, Stroop words, Stroop colour-word test). Additionally, participants had to show evidence of either subjective decline (score $\geq 25$ on the MACQ [32]) or evidence of decline (>3-point decline in MMSE score since wave 3 ) or evidence of consistent cognitive impairment over time (MMSE $\leq 24$ at waves 3 and 4).

All data derived from the health survey and cognitive testing as well as informant interview were collated into a spreadsheet case file for each participant. This case file (screen 2) automatically screened each participant for meeting criteria for any one of the following diagnoses: Diagnostic and Statistical Manual of Mental Disorders, Fifth Edition (DSM-5), major neurocognitive disorder (NCD); DSM-IV dementia; DSM-5 mild NCD; MCI; age-associated cognitive decline; age-associated memory impairment; DSM-IV amnestic disorder not otherwise specified; DSM-IV mild NCD; and DSM-IV other cognitive disorder. Major criteria for meeting most of these diagnoses were operationalised as any of the following: (1) concern of self or informant of significant cognitive decline (MACQ $\geq 25$ or Informant Questionnaire on Cognitive Decline in the Elderly $>3.31$ or history of dementia diagnosis); (2) substantial impairment on at least one cognitive domain relative to wave 4 normative data (cut-offs less than -2 SD for dementias, less than -1.5 SD for mild cognitive disorders); (3) interference with independence and instrumental activities of daily living (IADL; self-reported IADL impairment or Bayer IADL scale score $>3.11$ or informant-reported everyday cognitive difficulties); (4) not exclusively during delirium (cognitive changes of $>6$ months' duration, onset of cognitive changes preceding informant report of onset of deliriumlike symptoms); and (5) not due to another co-existing disorder (PHQ9 $<9$ and no reported history of schizophrenia or other psychosis). Those meeting criteria for one or more diagnoses $(n=368)$ were screened for case file review by a research neurologist. Diagnoses were made for 301 of these cases, of which 60 complex cases were selected for diagnostic consensus based on the following criteria: (1) comorbid depression, (2) other comorbid psychiatric conditions, (3) stroke and (4) DSM-5 major NCD without memory impairment. Following consensus diagnosis with a clinician specialising in psychiatry, the final diagnoses included 85 dementia/ major NCD, 196 mild cognitive disorders $(\mathrm{MCI} / \mathrm{mild}$ NCD), and 34 other mild or medically related cognitive disorders.

Clinically diagnosed MCI was based on the Petersen criteria at waves 1 and 2 [37], whereas the Winblad criteria [38] were used at waves 3 and 4. Clinically diagnosed dementia was based on the DSM-IV criteria [39] at all waves. At wave 4, there were 14 participants who were not interviewed but were known to have dementia on the basis of informant reports and medical records. Owing to the small number of individuals classified with dementia, participants with either MCI or dementia were grouped into a single $\mathrm{MCI} /$ dementia category.

\section{MCI-TB}

To complement the clinical diagnosis of MCI, a broader MCI-TB classification was applied to the entire PATH sample [40] at each wave on the basis of education-adjusted cognitive performance (Table 1). The PATH sample was first stratified by education $(0-12$ or $13+$ years $)$. Within each of these strata, individuals were classified as MCI-TB if they scored 1.5 SD below the mean on one or more of the psychometric tests used to assess the following cognitive domains: perceptual speed measured using the SDMT [33], episodic memory assessed using the immediate recall of the first trial of the California Verbal Learning Test (recall-immediate) [32], working memory measured using the Digit Span Backward from the Wechsler Memory Scale [41] and vocabulary assessed by the Spot-the-Word test [42].

\section{Data analysis}

All statistical analyses were performed using $\mathrm{R}$ version 3.1.2 software [43]. Because missing values can reduce power and introduce bias in the resulting estimates, missing values that were not attributable to attrition for the predictive variables used in the construction of the ANU-ADRI and the MCI-TB (see above) were imputed using an implementation of the random forests algorithm available in the 'missForest' package in $\mathrm{R}[44,45]$. This left 2078 individuals available for analysis. 


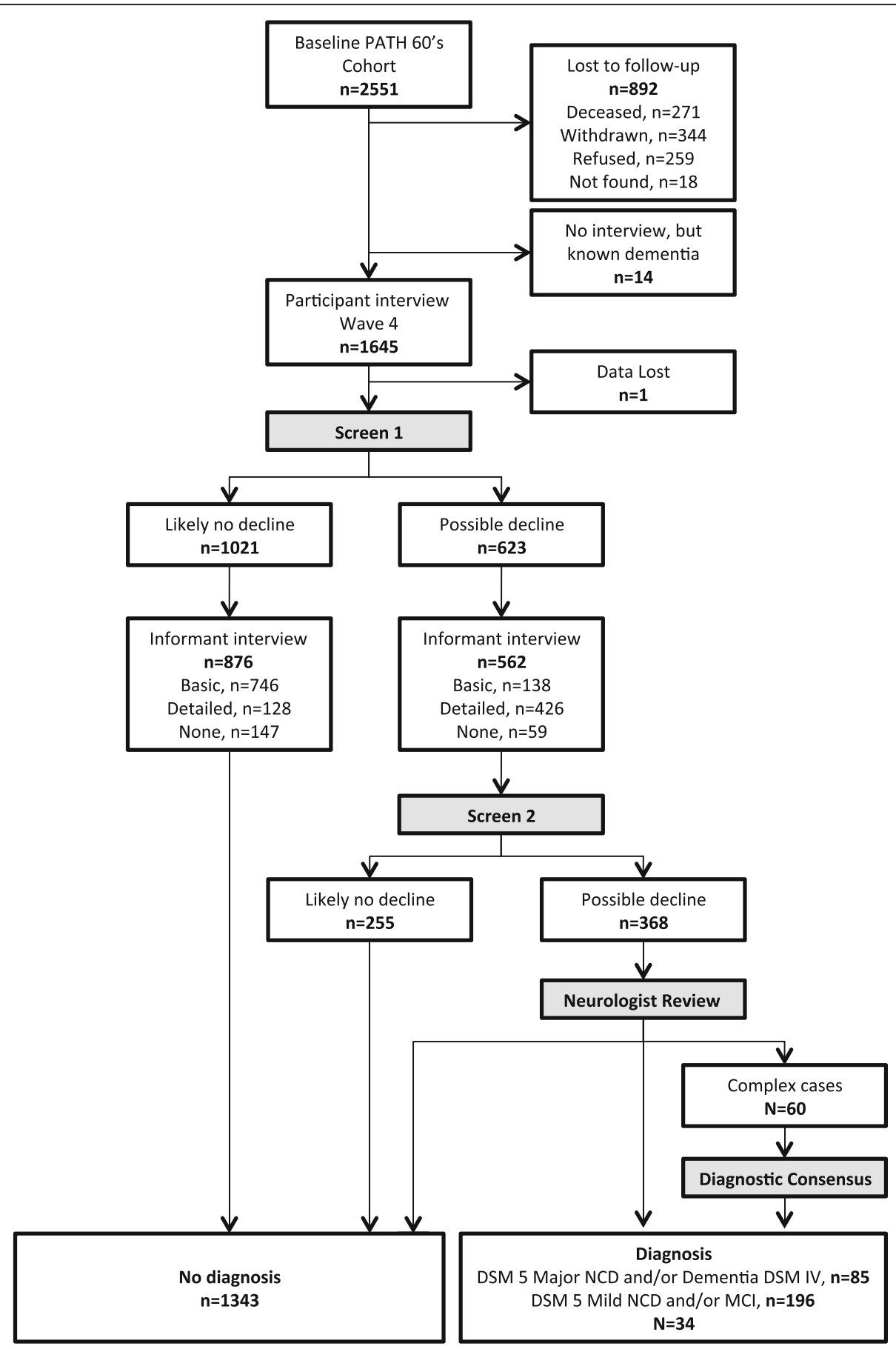

Fig. 1 Flowchart depicting the process of screening participants for mild cognitive disorders. DSM-IV Diagnostic and Statistical Manual of Mental Disorders, Fourth Edition; DSM-5 Diagnostic and Statistical Manual of Mental Disorders, Fifth Edition; MCI Mild cognitive impairment; NCD Neurocognitive disorder; PATH Personality and Total Health Through Life project

Additional file 2: Table $\mathrm{S} 2$ shows the proportion of missing variables for each variable.

We first evaluated the risk of progression from $\mathrm{CN}$ to $\mathrm{MCI} /$ dementia using Cox proportional hazards models with age as the time scale and the ANU-ADRI and EV-GRS included as predictor variables in the same model. The outcome of interest in these models was the time to first diagnosis of $\mathrm{MCI} /$ dementia, with those subjects who did not develop $\mathrm{MCI} /$ dementia at their last assessment right-censored. HRs and 95\% CIs were given for the time to $\mathrm{MCI} /$ dementia analysis. Concordance index (c-index) for the prediction of conversion from $\mathrm{NC}$ to 
Table 1 Characteristics of Personality and Total Health Through Life project cohort for waves 1-4

\begin{tabular}{|c|c|c|c|c|}
\hline & Wave 1 , estimate \pm SD & Wave 2 , estimate \pm SD & Wave 3 , estimate \pm SD & Wave 4 , estimate \pm SD \\
\hline$n$ & 2078 & 1798 & 1596 & 1337 \\
\hline Age, years & $63 \pm 1.5$ & $67 \pm 1.5$ & $71 \pm 1.5$ & $75 \pm 1.5$ \\
\hline Female sex, $n(\%)$ & 1009 (48.5) & $870(48.3)$ & 775 (48.6) & $651(48.7)$ \\
\hline Education & $14 \pm 2.8$ & - & - & - \\
\hline Wave 1 completers & $12.7 \pm 3.0$ & - & - & - \\
\hline Wave 2 completers & $13.1 \pm 2.7$ & - & - & - \\
\hline Wave 3 completers & $13.5 \pm 2.7$ & - & - & - \\
\hline Wave 4 completers & $14.2 \pm 2.6$ & - & - & - \\
\hline Immediate recall & $7.2 \pm 2.3$ & $7 \pm 2.2$ & $6.7 \pm 2.2$ & $5.4 \pm 1.9$ \\
\hline Digit Span Backward & $4.9 \pm 2.2$ & $5.1 \pm 2.2$ & $5.1 \pm 2.2$ & $5.3 \pm 2.2$ \\
\hline Spot-the-Word test & $52.0 \pm 6.0$ & $53 \pm 5.3$ & $53 \pm 5.1$ & $54 \pm 5$ \\
\hline SDMT & $50.0 \pm 9.7$ & $50 \pm 9.2$ & $48 \pm 9.2$ & $46 \pm 9.5$ \\
\hline ANU-ADRI & $9.4 \pm 5.9$ & - & - & - \\
\hline EV-GRS & $1.6 \pm 0.4$ & - & - & - \\
\hline \multicolumn{5}{|l|}{ Cognitive status, $n(\%)$} \\
\hline $\mathrm{MCl}$ & $23(1.1)$ & $28(1.6)$ & $35(2.2)$ & $103(7.7)$ \\
\hline Dementia & $0(0)$ & $0(0)$ & $7(0.44)$ & $37(2.7)$ \\
\hline $\mathrm{MCl}-\mathrm{TB}$ & $384(18.4)$ & $373(20.7)$ & $347(21.7)$ & $261(19.5)$ \\
\hline \multicolumn{5}{|l|}{ Attrition, $n(\%)$} \\
\hline Death & - & $57(2.7)$ & $54(2.5)$ & $94(5.8)$ \\
\hline Dropout & - & $280(13.5)$ & $167(9.3)$ & $329(20.6)$ \\
\hline
\end{tabular}

Abbreviations: ANU-ADRI Australian National University Alzheimer's Disease Risk Index, EV-GRS Explained variance-weighted genetic risk score, SDMT Symbol Digit Modalities Test, $M C I$ Mild cognitive impairment, MCI-TB Test-based mild cognitive impairment

$\mathrm{MCI} /$ dementia was calculated. Cox proportional hazards models were estimated using the 'survival' package in R.

To evaluate a more complex model of disease progression, MSMs were used to examine the association between the ANU-ADRI and EV-GRS and transitions between cognitive states. MSMs allow the modelling of competing risks and backward transitions between states (i.e., recovery) [46]. Hidden Markov models can be used to estimate misclassification error, and the effects of covariates can be allowed to vary by transition [46]. The MSMs used in this analysis modelled cognitive deterioration and cognitive recovery by allowing transitions and backward transitions between $\mathrm{CN}, \mathrm{MCI}$ or MCI-TB states. Backward transitions from dementia were not

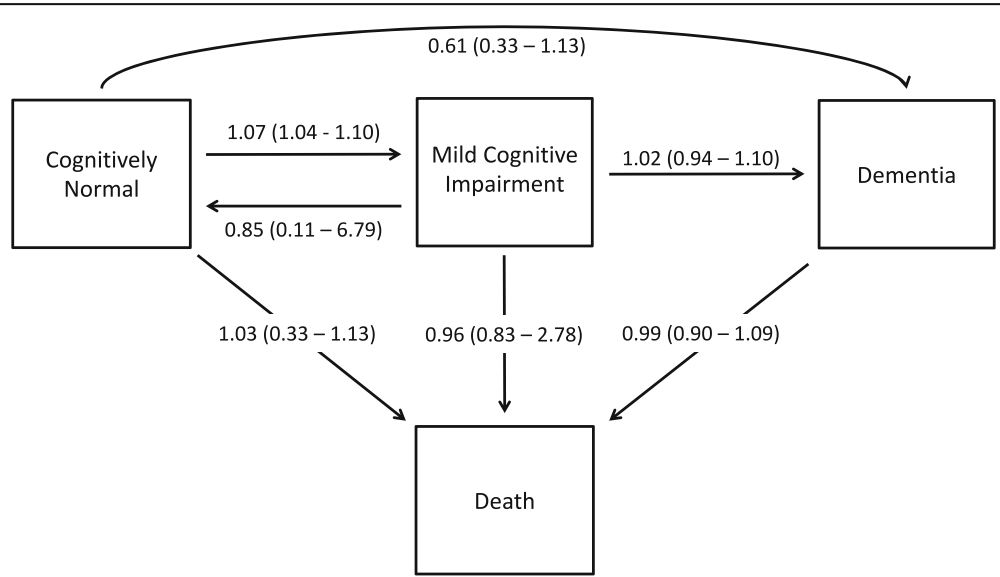

Fig. 2 A four-state model for possible transitions between cognitive states and death. HRs ( $95 \% \mathrm{Cls}$ ) for the effect of the Australian National University Alzheimer's Disease Risk Index on transitions between cognitively normal, mild cognitive impairment, dementia and death are shown. All estimates are from models adjusting for the explained variance-weighted genetic risk score 
allowed, whereas death was used as a fourth absorbing state (Fig. 2). Individuals with only a single observation (i.e., no recorded transitions) were excluded from the analysis $(n=204)$. Individuals lost to attrition were considered right-censored. The ANU-ADRI and the EV-GRS were included as covariates in the same model. Maximum likelihood estimates of parameters in the MSMs were obtained with the Broyden-Fletcher-Goldfarb-Shanno optimisation method. Normalisation was applied to the likelihood function to improve numerical stability. Because the likelihood is maximised using numerical methods, an input of initial values is required to start the search for a maximum. MSMs were fitted using 'msm' [46] in R, and multiple models were run using different sets of initial values to ensure the robustness of the parametric estimates. See Additional file 3 for more details on the structure of MSMs.

As a sensitivity analysis for the MCI-TB analysis, more stringent criteria were investigated with MCI-TB based on a score of $1.5 \mathrm{SD}$ below the mean on two or more of the above-mentioned psychometric tests. Additionally, we performed a complete case analysis to ensure that our imputation method was not biasing the observed results.

\section{Results}

\section{Demographics and other characteristics of the sample}

Baseline distributions of education, depression, sex, ANU-ADRI, raw cognitive tests scores and cognitive states at each wave for the PATH cohort are described in Table 1. Participants who completed all four waves of interviews had a higher level of education than participants who completed only the wave 1 interview $(t=-6.8, d f=331.3, p<0.001)$. Participants were followed for an average of 9.6 years (after accounting for loss due to attrition) and a total of 13.9 years. Group differences in the sub-indices of the ANU-ADRI between $\mathrm{CN}$ and either
$\mathrm{MCI} /$ dementia or MCI-TB can be found in Additional file 4: Table S3. The distribution of the ANU-ADRI and EV-GRS scores is shown in Fig. 3. As expected, the proportion of individuals classified as $\mathrm{MCI} /$ dementia increased over the course of the study, whereas the proportion of individuals classified as MCI-TB remained stable (Table 1). By wave $4,36 \%$ of the cohort had been lost to follow-up, with 57, 54 and 94 individuals deceased by waves 2, 3 and 4, respectively, and an additional 280, 267 and 329 individuals being lost to follow-up for other reasons (e.g., refusal, left catchment area) at waves 2, 3 and 4, respectively.

Between any two waves, a greater proportion of people transitioned from $\mathrm{CN}$ to MCI-TB (10.5\%) than from unimpaired to MCI (2.6\%), indicating that MCI-TB is a broader categorisation of cognitive impairment. A smaller proportion of individuals transitioned in the opposite direction-from MCI-TB to $\mathrm{CN}$ (31.3\%)-than from MCI to $\mathrm{CN}$ (44\%), indicating that MCI-TB is also a more stable category (Table 2).

\section{Cox proportional hazards models for incident $\mathrm{MCl}$}

A higher ANU-ADRI (indicating greater risk) score was associated with an increased risk of progression to both $\mathrm{MCI} /$ Dementia and MCI-TB (Table 3). The EV-GRS was not associated with progression to either $\mathrm{MCI} /$ Dementia or $\mathrm{MCI}$ TB. The interaction between the ANU-ADRI and the EVGRS was non-significant for the MCI/Dementia (HR 0.99 [95\% CI 0.96-1.01], $p=0.33$ ) and MCI-TB (HR 0.99 [95\% CI 0.98-1.01], $p=0.11$ ).

In the sensitivity analysis, using a more stringent MCITB criterion (scoring 1.5 SD below the mean on two or more tests) confirmed that the ANU-ADRI was associated an increased risk of progression from CN to MCI-TB (HR 1.08 [95\% CI 1.05-1.10], $p=<0.0001$ ). In the complete case analysis, the ANU-ADRI remained significant for both
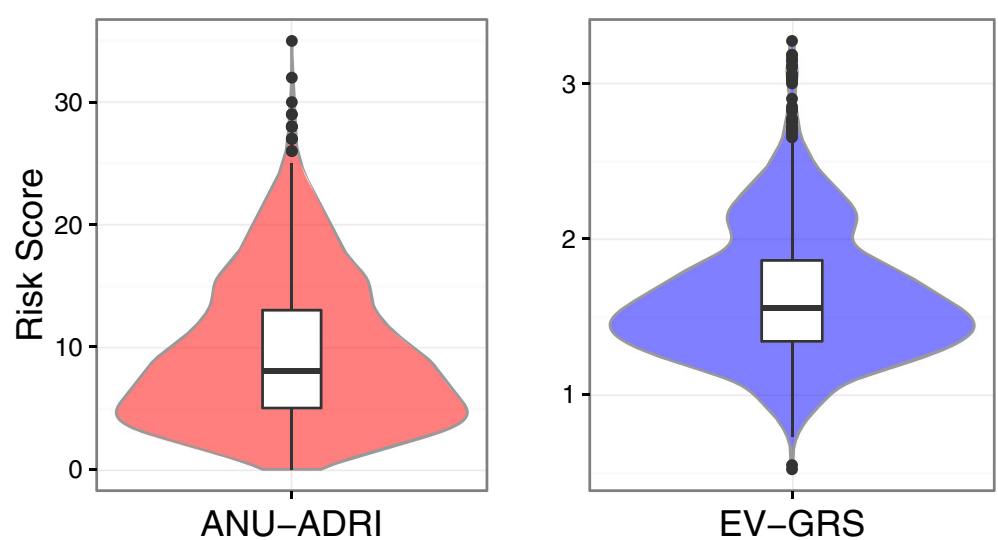

Fig. 3 Distribution of the Australian National University Alzheimer's Disease Risk Index (ANU-ADRI) and explained variance-weighted genetic risk score (EV-GRS) within the Personality and Total Health Through Life project cohort. The variable width of the violin plot indicates the probability density, and the box plot indicates the first, median and third quartiles of the ANU-ADRI and EV-GRS scores 
Table 2 Number of transitions between cognitively normal, mild cognitive impairment, dementia and test-based mild cognitive impairment during the length of the study

\begin{tabular}{|c|c|c|c|c|c|}
\hline \multicolumn{6}{|l|}{ To } \\
\hline From & $\mathrm{CN}$ & $\mathrm{MCl}$ & Dementia & Death & Censored \\
\hline \multicolumn{6}{|c|}{$\mathrm{MCl}$ and dementia } \\
\hline $\mathrm{CN}$ & 4459 (86.1\%) & $137(2.6 \%)$ & $32(0.6 \%)$ & $189(3.7 \%)$ & 359 (6.9\%) \\
\hline $\mathrm{MCl}$ & $40(48.2 \%)$ & $26(31.3 \%)$ & $6(7.2 \%)$ & $5(6 \%)$ & $6(7.2 \%)$ \\
\hline Dementia & $0(0 \%)$ & $0(0 \%)$ & $5(71.4 \%)$ & $2(28.6 \%)$ & $0(0 \%)$ \\
\hline Censored & $36(19.5 \%)$ & $3(1.6 \%)$ & $0(0 \%)$ & $8(4.3 \%)$ & $138(74.6 \%)$ \\
\hline \multicolumn{6}{|l|}{ MCl-TB } \\
\hline $\mathrm{CN}$ & 3403 (80.2\%) & $446(10.5 \%)$ & & $144(3.4 \%)$ & $249(5.9 \%)$ \\
\hline $\mathrm{MCl}$ & $321(31.3 \%)$ & $524(51.2 \%)$ & & $52(5.1 \%)$ & $127(12.4 \%)$ \\
\hline Censored & $28(15.1 \%)$ & $11(5.9 \%)$ & & $8(4.3 \%)$ & $138(74.6 \%)$ \\
\hline
\end{tabular}

the MCI/dementia (HR 1.06 [95\% CI 1.02-1.09], $p=0.001$ ) and MCI-TB (HR 1.036 [95\% CI 1.01-1.04], $p=0.007$ ) models.

\section{Multi-state models of transitions}

A higher ANU-ADRI score was associated with an increased risk of transitioning from $\mathrm{CN}$ to $\mathrm{MCI}$ or MCITB (Fig. 1, Table 4). The probability of transitioning from $\mathrm{CN}$ to either MCI or MCI-TB after 12 years for individuals scoring $1 \mathrm{SD}$ below the mean on the ANUADRI was $10 \%$, and for individuals scoring $1 \mathrm{SD}$ above the mean, the probability of transitioning was $20 \%$. A higher ANU-ADRI score was not associated with transitions from $\mathrm{CN}, \mathrm{MCI}$, dementia or MCI-TB to death; with transition from cognitive impairment to dementia; or with cognitive recovery from MCI or MCI-TB to CN. The EV-GRS was associated with an increased risk of transitioning from $\mathrm{CN}$ to dementia, with the probability of transitioning from $\mathrm{CN}$ to dementia for individuals scoring $1 \mathrm{SD}$ above the mean being $1.3 \%$. The interaction between the ANU-ADRI and the EV-GRS was

Table 3 Associations between the Australian National University Alzheimer's Disease Risk Index and explained variance-weighted genetic risk scores and cognitive impairment at waves 1-4

\begin{tabular}{lll}
\hline & MCl/dementia & MCI-TB \\
\hline ANU-ADRI $^{\mathrm{a}}, \mathrm{HR}(95 \% \mathrm{Cl})$ & $1.06(1.03-1.09)^{\mathrm{b}}$ & $1.04(1.02-1.50)^{\mathrm{b}}$ \\
EV-GRS ${ }^{\mathrm{b}}, \mathrm{HR}(95 \% \mathrm{Cl})$ & $1.14(0.98-1.33)$ & $1.04(0.96-1.12)$ \\
C-index (SE) & $0.61(0.03)$ & $0.56(0.01)$ \\
$\quad$ ANU-ADRI & $0.60(0.05)$ & $0.56(0.02)$ \\
$\quad$ EV-GRS & $0.53(0.05)$ & $0.51(0.02)$
\end{tabular}

Abbreviations: ANU-ADRI Australian National University Alzheimer's Disease Risk Index; C-index Concordance index; EV-GRS Explained variance-weighted genetic risk score

All estimates are derived from models adjusting for ANU-ADRI and EV-GRS

${ }^{\text {a }}$ Per unitary increase in ANU-ADRI

${ }^{\mathrm{b}} p<.001$

'Per SD increase in EV-GRS not significant for any of the transitions for either the MCI or MCI-TB models.

In the sensitivity analysis, using a more stringent MCI-TB criterion (Additional file 5: Table S4) confirmed that the ANU-ADRI was associated an increased risk of progression from $\mathrm{CN}$ to MCI-TB (HR 1.12 [95\% CI 1.07-1.17]). For the complete case analysis (Additional file 6: Table S5), the ANU-ADRI remained statistically significant for both the models for transition from $\mathrm{CN}$ to $\mathrm{MCI}$ (1.06 [1.02-1.09]) and from CN to MCI-TB (HR 1.05 [95\% CI 1.01-1.08]).

\section{Discussion}

To our knowledge, we report the first concurrent evaluation of a non-genetic risk score and a GRS in the risk of progression to MCI over a long period in a population-based cohort. As such, this study provides much-needed information on the utility of risk assessment tools in evaluating the risk of progression to $\mathrm{MCI}$ in the general population. Using Cox proportional hazards models, we found that a unitary increase in the ANU-ADRI at baseline was associated with $6 \%$ and $4 \%$ increased hazards of transitioning from $\mathrm{CN}$ to $\mathrm{MCI} / \mathrm{de}$ mentia and MCI-TB, respectively. Additionally, we used MSMs to extend the Cox proportional hazards models to account for backward transitions between cognitive states and the competing risks of death and dementia. We observed that a unitary change in the ANU-ADRI was associated with a $7 \%$ increased hazard of transitioning from $\mathrm{CN}$ to either MCI or MCI-TB. In contrast, the EVGRS was not associated with transition from $\mathrm{CN}$ to cognitive impairment, though it was associated with a $419 \%$ increased hazard of transitioning to dementia from $\mathrm{CN}$.

MSMs are well suited to analysing a more 'realistic' model of cognitive decline in which cognitive deterioration and recovery are modelled simultaneously in addition to misclassification, death and censoring. This is important in the examination of MCI because 
Table 4 HRs (95\% Cls) of the Australian National University Alzheimer's Disease Risk Index and explained variance-weighted genetic risk scores upon cognitive transition

\begin{tabular}{|c|c|c|c|c|}
\hline \multirow[t]{2}{*}{ Transition } & \multicolumn{2}{|c|}{$\mathrm{MCl}$ and dementia } & \multicolumn{2}{|l|}{$\mathrm{MCl}-\mathrm{TB}$} \\
\hline & $\overline{\mathrm{ANU}-\mathrm{ADRI}}{ }^{\mathrm{a}}$ & EV-GRS ${ }^{b}$ & $\overline{\text { ANU-ADRI }^{a}}$ & EV-GRS ${ }^{b}$ \\
\hline $\mathrm{CN}$ to $\mathrm{MCl}$ & $1.07(1.04-1.10)^{c}$ & $1.05(0.86-1.29)$ & $1.07(1.04-1.09)^{c}$ & $1.03(0.87-1.21)$ \\
\hline $\mathrm{CN}$ to dementia & $0.61(0.33-1.13)$ & $4.19(1.72-10.2)^{c}$ & & \\
\hline $\mathrm{CN}$ to death & $1.03(0.94-1.12)$ & $0.70(0.27-1.84)$ & $1.02(0.98-1.06)$ & $0.89(0.69-1.16)$ \\
\hline $\mathrm{MCl}$ to $\mathrm{CN}$ & $0.85(0.11-6.79)$ & $0.95(0-181.21)$ & $0.71(0.50-1.00)$ & $0.44(0.12-1.54)$ \\
\hline $\mathrm{MCl}$ to dementia & $1.02(0.94-1.10)$ & $1.19(0.76-1.85)$ & & \\
\hline $\mathrm{MCl}$ to death & $0.96(0.83-1.11)$ & $0.87(0.29-2.63)$ & $1.05(0.98-1.12)$ & $1.05(0.65-1.71)$ \\
\hline Dementia to death & $0.99(0.90-1.09)$ & $0.78(0.51-1.19)$ & & \\
\hline
\end{tabular}

Abbreviations: ANU-ADRI Australian National University Alzheimer's Disease Risk Index, EV-GRS Explained variance-weighted genetic risk score, CN Cognitively normal, MCl/dementia Mild cognitive impairment or dementia, MCI-TB Test-based mild cognitive impairment

All estimates are derived from models adjusting for the ANU-ADRI and EV-GRS

${ }^{\text {a }}$ Per unitary increase in ANU-ADRI

${ }^{b}$ Per SD increase in EV-GRS

${ }^{c} p<0.05$

pathological cognitive change is often not a linear progression from $\mathrm{CN}$ to $\mathrm{MCI}$ and finally to dementia; reversions from MCI back to $\mathrm{CN}$ are common, which was also observed in the PATH cohort [30, 47]. Individuals with a stable progression to $\mathrm{MCI}$ are more likely to progress to dementia than those with an unstable course or no diagnosis of MCI [47]. A higher ANU-ADRI score is associated both with an increased risk of transition to clinically diagnosed MCI and to MCI-TB, suggesting that it could be useful for assessing an individual's risk of developing MCI. Additionally, even in individuals who revert to $\mathrm{CN}$, the diagnosis of cognitive impairment may still have prognostic implications because these individuals have a greater likelihood of progressing to dementia or MCI than those who remain $\mathrm{CN}$ [47]. As such, individuals with a higher ANU-ADRI are more likely to revert to $\mathrm{MCI}$ or develop dementia in the future [15]. These results show that the ANU-ADRI may be used to measure risk reduction for clinically significant MCI as well as dementia, and it may have implications for secondary prevention of dementia. However, although the ANU-ADRI is strongly associated with the progression from $\mathrm{CN}$ to $\mathrm{MCI}$, its predictive ability was limited (c-index 0.60 for $\mathrm{MCI}$ and 0.56 for MCI-TB). This may be due to the relatively young age of the PATH cohort and consequently the small number of participants with MCI and the narrow age range of the sample. We expect that further validation of the ANU-ADRI in a slightly older cohort with a higher incidence of MCI or with a wider age range would show that the ANU-ADRI has greater predictive ability.

The ANU-ADRI has several strengths [4]. First, the ANU-ADRI is the only risk assessment tool that has not been developed by identifying risk factors through the analysis of a single cohort, and as such the predictive variables are not optimised to a particular study. The
ANU-ADRI also does not include any risk factors that require clinical assessments or laboratory tests.

The genetic risk score was observed to be associated with the transition from $\mathrm{CN}$ to dementia, but not with the transition from $\mathrm{CN}$ to $\mathrm{MCI}$ or from $\mathrm{MCI}$ to dementia. This lack of an association may be a result of the broad categorisation of MCI rather than MCI subtypes, such that it would have included participants with cognitive impairment that was not $\mathrm{MCI}$ due to $\mathrm{AD}[48,49]$. This may also explain the reduced risk associated with both MCI and MCI-TB in our sensitivity analysis. Unfortunately, owing to the small number of participants with MCI in the PATH cohort, further subgroup analysis would likely be underpowered to detect an effect. However, it should be noted that most dementia cases are associated with mixed pathologies rather than singular pathologies, suggesting that an AD GRS would be associated with both amnestic and non-amnestic MCI [50].

Researchers in previous studies have investigated the association of AD GRS with MCI. In 3605 participants (360 MCI, 191 dementia), an AD GRS composed of $A P O E+19$ LOAD GWAS variants was associated with an increased risk of incident $\mathrm{MCI}$ and nominally associated with amnestic and non-amnestic cases [9]. In a second study of 2674 participants (347 MCI, 132 LOAD), a GRS composed of $A P O E+$ nine LOAD GWAS variants was associated with progression from $\mathrm{CN}$ to $\mathrm{MCI} / \mathrm{LOAD}$ [10]. Lack of replication in this study could be due to younger and fewer cognitively impaired participants. Furthermore, inclusion of additional AD risk loci that were identified to be nominally significant in relation to AD in GWASs may identify a stronger association [8].

Limitations of our study include the relatively high level of education of the PATH cohort [16]. Also, the ethnicity distribution in the PATH cohort is predominately Caucasian, potentially limiting the 
generalisability of the results of this study to other ethnicities, and biomarkers of AD were not available (e.g., cerebrospinal fluid, amyloid- $\beta$ ). Not all the predictive variables for the ANU-ADRI were available in PATH, suggesting that the present study may underestimate the sensitivity of this tool in predicting individuals who are at risk of developing cognitive impairment. However, the validation studies also included a subset of the variables contributing to the ANU-ADRI [15].

Study strengths included the large population-based sample with high retention rates and 12 years of followup data. The PATH cohort was recruited from a narrow age band, reducing the impact of age differences on the findings. This is particularly important because age has the largest weighting of risk factors in the ANU-ADRI. Finally, the conservative clinical classifications of MCI/ dementia, based on a thorough clinical assessment and consensus diagnosis by clinicians using published criteria, were complemented by a broader classification of MCI (MCI-TB).

\section{Conclusions}

Higher ANU-ADRI scores are associated with increased risk of progressing from $\mathrm{CN}$ to $\mathrm{MCI}$. These results complement previous evidence that the ANU-ADRI is predictive of $A D$ and dementia [15]. In comparison, a GRS comprising the main $\mathrm{AD}$ genes was associated with the development of dementia but was not associated with the risk of developing MCI. These results provide further support for using the ANU-ADRI for population-level strategies, individual patient assessment, and for informing intervention and treatment strategies aimed at delaying or preventing dementia.

\section{Additional files}

Additional file 1: Table S1. LOAD risk SNPs used in this study. (DOCX $89 \mathrm{~kb}$ )

Additional file 2: Table S2. Proportion of missing data that was imputed for ADRI sub-indices and cognitive variables. (DOCX $93 \mathrm{~kb}$ )

Additional file 3: Supplementary methods. Further detail on multi-state models. (DOCX $93 \mathrm{~kb}$ )

Additional file 4: Table S3. Differences between NC and individuals classified as $\mathrm{MCl} /$ dementia or $\mathrm{MCl}$-TB at any wave for the ANU-ADRI, ANU-ADRI sub-indices and the EV-GRS. (DOCX $91 \mathrm{~kb}$ )

Additional file 5: Table S4. Hazard ratios ( $95 \% \mathrm{Cls}$ ) of the ANU-ADRI and EV-GRS scores upon cognitive transition in the MCl-TB sensitivity analysis using a more stringent criterion of MCI-TB (scoring 1.5 SD below the mean on two or more tests). (DOCX $51 \mathrm{~kb}$ )

Additional file 6: Table S5. Hazard ratios (95\% Cls) of the ANU-ADRI and EV-GRS scores upon cognitive transition in the complete case sensitivity analysis. (DOCX $65 \mathrm{~kb}$ )

\section{Abbreviations}

AD: Alzheimer's disease; ANU-ADRI: Australian National University Alzheimer's Disease Risk Index; APOE: Apolipoprotein E; BMI: Body mass index; c-index: Concordance index; CN: Cognitively normal; DSM-5: Diagnostic and Statistical Manual of Mental Disorders, Fifth Edition; DSM-IV: Diagnostic and Statistical Manual of Mental Disorders, Fourth Edition; EV-GRS: Explained variance-weighted genetic risk score; GRS: Genetic risk score;

GWAS: Genome-wide association study; IADL: Instrumental activities of daily living; LOAD: Late-onset Alzheimer's disease; MACQ: Memory and Cognition Questionnaire; MAF: Minor allele frequency; MCl: Mild cognitive impairment; MCl-TB: Test-based mild cognitive impairment; MMSE: Mini Mental State Examination; MSM: Multi-state model; NCD: Neurocognitive disorder; NHMRC: National Health and Medical Research Council; PATH: Personality and Total Health Through Life project; PHQ-9: Patient Health Questionnaire SDMT: Symbol Digit Modalities Test; SNP: Single-nucleotide polymorphism

\section{Acknowledgements}

We thank the participants in the PATH study, Peter Butterworth, Andrew Mackinnon, Anthony Jorm, Bryan Rodgers, Helen Christensen, Patricia Jacomb and Karen Mawell.

\section{Funding}

The study was supported by the Dementia Collaborative Research Centres and National Health and Medical Research Council (NHMRC) grants 973302 179805, 1002160 and 1002560. JIV was supported by the Eccles Scholarship in Medical Sciences, the Fenner Merit Scholarship and The Australian National University High Degree Research scholarships. NC is funded by Research Fellowship number 12010227. KJA is funded by NHMRC Research Fellowship number 1002560. The funders had no role in study design, data collection and analysis, decision to publish, or preparation of the manuscript.

\section{Availability of data and materials}

Data are available from the Personality and Total Health (PATH) Through Life Governance Committee for researchers who meet the criteria for access to confidential data. Contact Email: path@anu.edu.au. Web address: http://crahw.anu.edu.au/research/projects/personality-total-health-paththrough-life.

\section{Authors' contributions}

SJA and KJA conceived of and designed the study. SJA, JIV and KJA contributed to the design of the analysis. SJA analysed the data and wrote the first draft of the manuscript. SJA, RE, JIV, NC, SE and KJA contributed to the writing of the manuscript. RE scrubbed and maintained research data. All authors have read and confirm that they meet International Committee of Medical Journal Editors criteria for authorship. All authors read and approved the final manuscript.

Authors' information

Not applicable.

\section{Competing interests}

The authors declare that they have no competing interests.

\section{Consent for publication}

Not applicable.

Ethics approval and consent to participate

Written informed consent was obtained from all participants. This study was approved by the human research ethics committee of The Australian National University.

\section{Author details}

'John Curtin School of Medical Research, Australian National University, Canberra, Australia. ${ }^{2}$ Centre for Research on Ageing, Health and Wellbeing, Research School of Population Health Australian National University, The Australian National University Florey, Building 54, Mills Road, Acton ACT 2601, Canberra, Australia. ${ }^{3}$ Universidad del Norte, Barranquilla, Colombia.

${ }^{4}$ Neuroscience Research Group, University of Antioquia, Medellin, Colombia. 
Received: 10 October 2016 Accepted: 1 February 2017 Published online: 04 March 2017

\section{References}

1. Stephan BCM, Kurth T, Matthews FE, Brayne C, Dufouil C. Dementia risk prediction in the population: are screening models accurate? Nat Rev Neurol. 2010:6:318-26.

2. Tang EYH, Harrison SL, Errington L, Gordon MF, Visser PJ, Novak G, et al. Current developments in dementia risk prediction modelling: an updated systematic review. PLoS One. 2015;10:e0136181.

3. Prince $M$, Bryce $R$, Albanese E, Wimo A, Ribeiro W, Ferri CP. The global prevalence of dementia: a systematic review and metaanalysis. Alzheimers Dement. 2013;9:63-75.e2.

4. Anstey KJ, Eramudugolla R, Dixon RA. Contributions of a risk assessment approach to the prevention of Alzheimer's disease and dementia. J Alzheimers Dis. 2014;42 Suppl 4:S463-73.

5. Pankratz VS, Roberts RO, Mielke MM, Knopman DS, Jack CR, Geda YE, et al. Predicting the risk of mild cognitive impairment in the Mayo Clinic Study of Aging. Neurology. 2015:84:1433-42.

6. Karch CM, Goate AM. Alzheimer's disease risk genes and mechanisms of disease pathogenesis. Biol Psychiatry. 2015;77:43-51.

7. Verhaaren BFJ, Vernooij MW, Koudstaal PJ, Uitterlinden AG, van Duijn CM, Hofman A, et al. Alzheimer's disease genes and cognition in the nondemented general population. Biol Psychiatry. 2013;73:429-34

8. Escott-Price V, Sims R, Bannister C, Harold D, Vronskaya M, Majounie E, et al. Common polygenic variation enhances risk prediction for Alzheimer's disease. Brain. 2015;138:3673-84.

9. Adams HHH, de Bruijn RFAG, Hofman A, Uitterlinden AG, van Duijn CM, Vernooij MW, et al. Genetic risk of neurodegenerative diseases is associated with mild cognitive impairment and conversion to dementia. Alzheimers Dement. 2015;11:1277-85.

10. Carrasquillo MM, Crook JE, Pedraza O, Thomas CS, Pankratz VS, Allen M, et al. Late-onset Alzheimer's risk variants in memory decline, incident mild cognitive impairment and Alzheimer's disease. Neurobiol Aging. 2015:36:60-7.

11. Seshadri S, Fitzpatrick AL, Ikram MA, DeStefano AL, Gudnason V, Boada M, et al. Genome-wide analysis of genetic loci associated with Alzheimer disease. JAMA. 2010;303:1832-40

12. Rodríguez-Rodríguez E, Sánchez-Juan P, Vázquez-Hiquera JL, Mateo I, Pozueta A, Berciano J, et al. Genetic risk score predicting accelerated progression from mild cognitive impairment to Alzheimer's disease. J Neural Transm (Vienna). 2013;120:807-12.

13. Lacour A, Espinosa A, Louwersheimer E, Heilmann S, Hernández I, Wolfsgruber S, et al. Genome-wide significant risk factors for Alzheimer's disease: role in progression to dementia due to Alzheimer's disease among subjects with mild cognitive impairment. Mol Psychiatry. 2017;22:153-60.

14. Anstey KJ, Cherbuin N, Herath PM. Development of a new method for assessing global risk of Alzheimer's disease for use in population health approaches to prevention. Prev Sci. 2013;14:411-21.

15. Anstey KJ, Cherbuin N, Herath PM, Qiu C, Kuller LH, López OL, et al. A selfreport risk index to predict occurrence of dementia in three independent cohorts of older adults: the ANU-ADRI. PLoS One. 2014:9:e86141.

16. Anstey KJ, Christensen H, Butterworth P, Easteal S, Mackinnon A, Jacomb T, et al. Cohort profile: the PATH through life project. Int J Epidemiol. 2012;41:951-60.

17. National Health and Medical Research Council (NHMRC). Australian alcohol guidelines: health risks and benefits. Canberra: NHMRC; 2001.

18. Martin A, Rief W, Klaiberg A, Braehler E. Validity of the Brief Patient Health Questionnaire Mood Scale (PHQ-9) in the general population. Gen Hosp Psychiatry. 2006;28:71-7.

19. Bielak AAM, Cherbuin N, Bunce D, Anstey KJ. Preserved differentiation between physical activity and cognitive performance across young, middle, and older adulthood over 8 years. J Gerontol B Psychol Sci Soc Sci. 2014;69:523-32.

20. Naj AC, Jun G, Beecham GW, Wang LS, Vardarajan BN, Buros J, et al. Common variants at MS4A4/MS4A6E, CD2AP, CD33 and EPHA1 are associated with late-onset Alzheimer's disease. Nat Genet. 2011;43:436-41.

21. Hollingworth P, Harold D, Sims R, Gerrish A, Lambert JC, Carrasquillo MM, et al. Common variants at ABCA7, MS4A6A/MS4A4E, EPHA1, CD33 and CD2AP are associated with Alzheimer's disease. Nat Genet. 2011;43:429-35.
22. Harold D, Abraham R, Hollingworth P, Sims R, Gerrish A, Hamshere ML, et al. Genome-wide association study identifies variants at CLU and PICALM associated with Alzheimer's disease. Nat Genet. 2009;41:1088-93.

23. Lambert JC, Heath S, Even G, Campion D, Sleegers K, Hiltunen M, et al. Genome-wide association study identifies variants at CLU and CRI associated with Alzheimer's disease. Nat Genet. 2009:41:1094-9.

24. Lambert JC, Ibrahim-Verbaas CA, Harold D, Naj AC, Sims R, Bellenguez C, et al. Meta-analysis of 74,046 individuals identifies 11 new susceptibility loci for Alzheimer's disease. Nat Genet. 2013:45:1452-8.

25. Andrews SJ, Das D, Cherbuin N, Anstey KJ, Easteal S. Association of genetic risk factors with cognitive decline: the PATH through life project. Neurobiol Aging. 2016:41:150-8.

26. Andrews SJ, Das D, Anstey KJ, Easteal S. Late onset Alzheimer's disease risk variants in cognitive decline: the PATH Through Life Study. doi:10.3233/ JAD-160774.

27. Jorm AF, Mather KA, Butterworth P, Anstey KJ, Christensen H, Easteal S. $A P O E$ genotype and cognitive functioning in a large age-stratified population sample. Neuropsychology. 2007;21:1-8.

28. Che R, Motsinger-Reif AA. Evaluation of genetic risk score models in the presence of interaction and linkage disequilibrium. Front Genet. 2013:4:138.

29. Anstey KJ, Cherbuin N, Christensen H, Burns R, Reglade-Meslin C, Salim A, et al. Follow-up of mild cognitive impairment and related disorders over four years in adults in their sixties: the PATH Through Life Study. Dement Geriatr Cogn Disord. 2008;26:226-33.

30. Anstey KJ, Cherbuin N, Eramudugolla R, Sargent-Cox K, Easteal S, Kumar R, et al. Characterizing mild cognitive disorders in the young-old over 8 years: prevalence, estimated incidence, stability of diagnosis, and impact on IADLs. Alzheimers Dement. 2013:9:640-8.

31. Folstein MF, Folstein SE, McHugh PR. "Mini-mental state": a practical method for grading the cognitive state of patients for the clinician. J Psychiatr Res. 1975;12:189-98.

32. Delis DC, Kramer JH, Kaplan E, Ober BA. California Verbal Learning Test. San Antonio: Psychological Corporation; 1987.

33. Smith A. Symbol Digit Modalities Test: manual. Los Angeles: Western Psychological Corporation; 2002.

34. Tiffin J. Purdue Pegboard examiner manual. Revth ed. Chicago: Science Research Associates; 1968.

35. Anstey KJ, Dear K, Christensen H, Jorm AF. Biomarkers, health, lifestyle, and demographic variables as correlates of reaction time performance in early, middle, and late adulthood. Q J Exp Psychol A. 2005;58:5-21.

36. Morris JC. The Clinical Dementia Rating (CDR): current version and scoring rules. Neurology. 1993;43:2412-4.

37. Petersen RC, Smith GE, Waring SC, Ivnik RJ, Tangalos EG, Kokmen E. Mild cognitive impairment: clinical characterization and outcome. Arch Neurol. 1999;56:303-8.

38. Winblad B, Palmer K, Kivipelto M, Jelic V, Fratiglioni L, Wahlund LO, et al. Mild cognitive impairment - beyond controversies, towards a consensus: report of the International Working Group on Mild Cognitive Impairment. J Intern Med. 2004:256:240-6.

39. American Psychiatric Association. Diagnostic criteria for dementia of the Alzheimer's type. In: Diagnostic and statistical manual of menta disorders (DSM-IV). 4th ed. Washington, DC: American Psychiatric Press; 1994. p. 143-7.

40. Dixon RA, DeCarlo CA, MacDonald SWS, Vergote D, Jhamandas J, Westaway D. APOE and COMT polymorphisms are complementary biomarkers of status, stability, and transitions in normal aging and early mild cognitive impairment. Front Aging Neurosci. 2014;6:236.

41. Wechsler D. A standardized memory scale for clinical use. J Psychol 1945:19:87-95.

42. Baddeley A, Emslie H, Nimmo-Smith I. The Spot-the-Word test: a robust estimate of verbal intelligence based on lexical decision. Br J Clin Psychol. 1993:32:55-65.

43. Ihaka R, Gentleman R. R: a language for data analysis and graphics. J Comput Graph Stat. 1996;5:299-314.

44. Stekhoven DJ, Bühlmann P. MissForest—non-parametric missing value imputation for mixed-type data. Bioinformatics. 2012;28:112-8.

45. Waljee AK, Mukherjee A, Singal AG, Zhang Y, Warren J, Balis U, et al Comparison of imputation methods for missing laboratory data in medicine. BMJ Open. 2013;3:e002847.

46. Jackson C. Multi-state models for panel data: the msm package for $\mathrm{R}$. J Stat Soft. 2011;38(8):1-29. 
47. Roberts RO, Knopman DS, Mielke MM, Cha RH, Pankratz VS, Christianson TJH, et al. Higher risk of progression to dementia in mild cognitive impairment cases who revert to normal. Neurology. 2014;82:317-25.

48. Petersen RC, Negash S. Mild cognitive impairment: an overview. CNS Spectr. 2008;13:45-53.

49. Albert MS, DeKosky ST, Dickson D, Dubois B, Feldman HH, Fox NC, et al. The diagnosis of mild cognitive impairment due to Alzheimer's disease: recommendations from the National Institute on Aging-Alzheimer's Association workgroups on diagnostic guidelines for Alzheimer's disease. Alzheimers Dement. 2011;7:270-9.

50. Kawas CH, Kim RC, Sonnen JA, Bullain SS, Trieu T, Corrada MM. Multiple pathologies are common and related to dementia in the oldest-old: The 90+ Study. Neurology. 2015;85:535-42.

Submit your next manuscript to BioMed Central and we will help you at every step:

- We accept pre-submission inquiries

- Our selector tool helps you to find the most relevant journal

- We provide round the clock customer support

- Convenient online submission

- Thorough peer review

- Inclusion in PubMed and all major indexing services

- Maximum visibility for your research

Submit your manuscript at www.biomedcentral.com/submit
Biomed Central 